\title{
Direct Observation of Heterogeneous Formation of Amyloid Spherulites in Real-time by Super- resolution Microscopy
}

Nikos Hatzakis ( $\square$ hatzakis@nano.ku.dk)

University of Copenhagen

\section{Min Zhang}

University of Copenhagen, Denmark https://orcid.org/0000-0002-2797-5049

Henrik Pinholt

University of Copenhagen

\section{Xin Zhou}

University of Copenhagen

\section{Søren Bohr}

University of Copenhagen,

\section{Luca Banetta}

Polytechnic University of Turin

\author{
Alessio Zaccone \\ University of Milan \\ Vito Foderà \\ University of Copenhagen
}

\section{Article}

Keywords: Super-resolution imaging, protein aggregation, spherulites, real-time, REPLOM.

Posted Date: February 15th, 2022

DOI: https://doi.org/10.21203/rs.3.rs-1338225/v1

License: (c) (i) This work is licensed under a Creative Commons Attribution 4.0 International License.

Read Full License

Version of Record: A version of this preprint was published at Communications Biology on August 20th, 2022. See the published version at https://doi.org/10.1038/s42003-022-03810-1. 


\section{Direct Observation of Heterogeneous Formation of}

\section{Amyloid Spherulites in Real-time by Super-}

3 resolution Microscopy

4 Min Zhang ${ }^{1}$, Henrik D. Pinholt ${ }^{1}$, Xin Zhou ${ }^{2}$, Søren S.-R. Bohr ${ }^{1}$, Luca Banetta ${ }^{3}$, Alessio Zaccone,

5 Vito Foderă ${ }^{2, *}$ and Nikos S. Hatzakis ${ }^{1,5, *}$.

$6 \quad{ }^{1}$ Department of Chemistry, Faculty of Science, University of Copenhagen, Denmark

$7 \quad{ }^{2}$ Department of Pharmacy, Faculty of Health and Medical Sciences, University of Copenhagen,

8 Denmark

$9 \quad{ }^{3}$ Department of Applied Science and Technology, Polytechnic University of Turin, Italy

$10 \quad{ }^{4}$ Department of Physics, University of Milan, Italy

$11{ }^{5}$ NovoNordisk centre for Protein research, Faculty of Health and Medical Sciences University of

12 Copenhagen, Denmark

13 KEYWORDS: Super-resolution imaging, protein aggregation, spherulites, real-time, REPLOM.

14 
17 The misfolding of proteins and their aggregation in the form of fibrils or amyloid-like spherulites 18 are involved in a spectrum of pathological abnormalities. Our current understanding of protein 19 amyloid aggregation mechanisms has primarily relied on the use of spectrometric methods to 20 determine the average growth rates and diffraction limited microscopes with low temporal 21 resolution to observe the large-scale morphologies of intermediates. We developed a REal-time 22 kinetics via binding and Photobleaching LOcalisation Microscopy (REPLOM) super-resolution 23 method to directly observe and quantify the existence and abundance of diverse aggregate 24 morphologies of the model system insulin, below the diffraction limit and extract their 25 heterogeneous growth kinetics. Our results revealed that even the growth of a microscopically 26 identical aggregates, e.g. amyloid spherulites, may follow distinct pathways. Specifically, 27 spherulites do not exclusively grow isotropically but, surprisingly, may also grow anisotropically, 28 following similar pathways as reported for minerals and polymers. Combining our technique with 29 machine learning approaches, we associated growth rates to specific morphological transitions and 30 provided energy barriers and the energy landscape at the level of single aggregate morphology.

31 Our unifying framework for the detection and analysis of spherulite growth can be extended to 32 other self-assembled systems characterized by a high degree of heterogeneity, disentangling the 33 broad spectrum of diverse morphologies at the single-molecule level. 
37 Protein misfolding is a hallmark of a number of devastating conditions, such as Alzheimer's and

38 Parkinson's disease ${ }^{1-3}$. Moreover, insulin aggregates have been found deposited at diabetic 39 patients' insulin injection sites ${ }^{4}$. As one of the important aggregates, amyloid spherulites have 40 been found in the brain tissues in connection with the onset and progression of Alzheimer's 41 disease ${ }^{2,3}$. In addition, they may also present opportunities to develop advanced materials for drug 42 delivery ${ }^{5}$. Spherulites ranging from a few micrometers to several millimeters in diameter can form 43 both in vivo and in vitro and are observed during the aggregation of multiple proteins including 44 insulin, that we used as a model system here ${ }^{3,6,7}$. These aggregates are characterized by a 45 fascinating core-shell morphology and seem to be the result of a general self-assembly process that 46 is common to metal alloys ${ }^{8}$, minerals ${ }^{9}$, and polymers ${ }^{10,11}$. While we have a solid understanding 47 of the fibrillar growth kinetics ${ }^{12}$, the mechanisms of the formation and growth of spherulites is 48 still limited ${ }^{13-15}$.

49 The studies of protein spherulite formation primarily rely on spectrometric evidence for their 50 average growth rates ${ }^{13,16}$. Considering that a high heterogeneity of aggregate populations may 51 present within the same self-assembly reaction ${ }^{17}$, bulk methods provide limited information on 52 the aggregation kinetics of individual species, in the form of either fibrils or spherulites, averaging 53 the effect of the morphological heterogeneity of the aggregate population. Fluorescent microscopy, 54 as an intuitive and non-invasive method, has been used more and more in the area of protein 55 aggregation to record the growth intermediates ${ }^{15}$ and to observe the final structures ${ }^{18}$. While the 56 direct observation of fibril growth and time lapses of spherulite growth with temporal resolution 57 of minutes was recently reported for $\mathrm{A} \beta$ peptides 15,19-21, the kinetic analysis of individual 58 aggregates mainly focuses on fibrils, which challenges the evaluation of the kinetics of the multiple 59 and concurrent pathways. Meanwhile, due to the complex structures of spherulites, the resolution 60 of diffraction-limited microscopy such as TIRFM or confocal microscopy is not sufficient to 61 decipher the details of spherulites 15,22,23. Super-resolution methods surpass the diffraction 62 limitations, albeit often provide snapshots of the growth or the final morphology of fibrils ${ }^{24-26}$ 63 offering limited information on the temporal development of diverse structures. 
Here we developed a method named Real-time kinetics via binding and Photobleaching 65 Localisation Microscopy (REPLOM) to directly observe the formation of individual protein amyloid structures using human insulin (HI) as a model system. Attaining real-time videos of the

67 spherulite growth process allowed to reconstruct the super-resolution images of the spherulites and 68 their growth kinetics. Using homemade software based on Euclidian minimum spanning tree and machine learning clustering ${ }^{27-31}$, we quantitatively associated the growth rates to specific morphological transitions during growth, eventually extracting detailed energy barriers and, thus, the energy landscape for each type of aggregation morphology. Our data on astigmatism-based 3D direct stochastic optical reconstruction microscopy (dSTORM) ${ }^{32}$, spinning disk confocal 73 microscopy ${ }^{33}$, and scanning electron microscopy (SEM) confirm that the presence of

74 heterogeneous structures is not artefact of our method. Our combined results allowed us to differentiate among the different species in solution and decipher the nature, morphology and abundance of individual spherulites at different growth stages. Surprisingly, we found that HI spherulite growth is not exclusively isotropic and may occur anisotropically. We anticipate that the framework presented here will serve as a unique and generic methodology for the simultaneous detection and analysis of multiple species within a single self-assembly reaction. In the specific case of protein systems, the aggregation of which is related to degenerative diseases, our approach provides a platform for connecting kinetics, morphological transitions, and structure and further aid our understanding on interventions against degenerative diseases.

\section{Results and Discussion}

\section{Direct observation of diverse structures of HI spherulites by 3D dSTORM, SEM and}

\section{5 spinning disk microscopy}

86 We thermally induced insulin amyloid aggregation using an established protocol ${ }^{34}$ and examined

87 the bulk kinetics by detecting the fluorescence of the amyloid-sensitive dye Thioflavin T (ThT) 88 and the turbidity signal (Figure S1a). The kinetics traces at incubation temperature of $60{ }^{\circ} \mathrm{C}$, show 89 the classical three-step profile, with the reaction reaching completion after only 3-4 hours. The 90 turbidity and ThT signal perfectly overlapped, suggesting that the aggregation reaction was 91 entirely of an amyloid-like origin ${ }^{35}$. Cross-polarized microscopy recordings of the characteristic 92 Maltese cross, indicating spherulite formation under these conditions ${ }^{7}$ (Figure S2). However, 
93 standard analysis of the bulk ThT signal was unable to provide information on the morphological

94 transition occurring during the reaction.

95 To observe directly and with high-resolution the diverse structures of insulin aggregates, we 96 combined the insights obtained from SEM and 3D dSTORM. Using 3D dSTORM allowed us to 97 extend beyond diffraction-limited imaging by TIRF microscopy, which may mask spherulite shape 98 and growth directionality ${ }^{32}$ (Figure 1). Recordings at incubation times between 0.5 to 4 hours 99 points (see methods) provided direct recordings of the diverse early species that can co-exist at the 100 same incubation time. We found spherical-like protein condensates of approximately $200 \mathrm{~nm}$ in 101 diameter formed after 0.5 hours, while, a linear pattern was observed with incubation times ranging 102 between $0.5-1 \mathrm{~h}$. Surprisingly, the recordings beyond the diffraction limit revealed that at longer 103 incubation times the commonly observed spherulites were found to co-exist in the mixture with 104 anisotropically grown structures (Figure 1, Figure S4, S5c and S5d).

105 The fact that both SEM and 3D dSTORM methods identified the same particle morphology 106 supports this not to be an artifact of fluorescence microscopy, fluorophore labeling (Figure S1b), 107 sample drying for SEM imaging (Figure 1). Note however that depending on conditions the 108 distribution of morphologies may vary slightly consistent with earlier reporting of electrostatic 109 effect for $A \beta-(1-40)^{15}$ (see Figure S5f). Extending beyond the diffraction limit suggest that protein 110 spherulite growth may diverge from isotropically grown in space ${ }^{7,36}$, and proceed in a preferential 111 direction.

112 The density plots created with 3D dSTORM (Figure 1) clearly showed that the core had a much 113 higher density than the branching parts, consistent with previous suggestions of the existence of a 114 low-density corona in spherulite structures ${ }^{7,37}$. The formation of the high-density cores appears to 115 indicate the nucleation point, with the subsequent linear-like elongation and branching of slender 116 threadlike fibrils resembling crystalline growth ${ }^{38,39}$. This is consistent with the recently proposed 117 initial protein condensation process ${ }^{40}$, and further growth is determined by tight fibril packing, 118 which forces the biomolecular assembly to occur anisotropically along one specific direction. 119 Delineating this however would require additional experiment and is beyond the scope of this 120 study. The directly observed anisotropy challenges the isotropic spherulite growth, for which the 121 process occurs via the formation of a radiating array of fiber crystallites, but it is observed in the 
case of crystalline-coil block copolymer spherulites ${ }^{41}$. The origin of such anisotropy might be due

123 to the occurrence of secondary and heterogeneous nucleation at the aggregate surface ${ }^{20,42}$, with

124 different binding efficiencies depending on the aggregate areas. While the data in Figure 1 would 125 be consistent with the secondary nucleation, deciphering this with additional data falls beyond the 126 scope of this work.

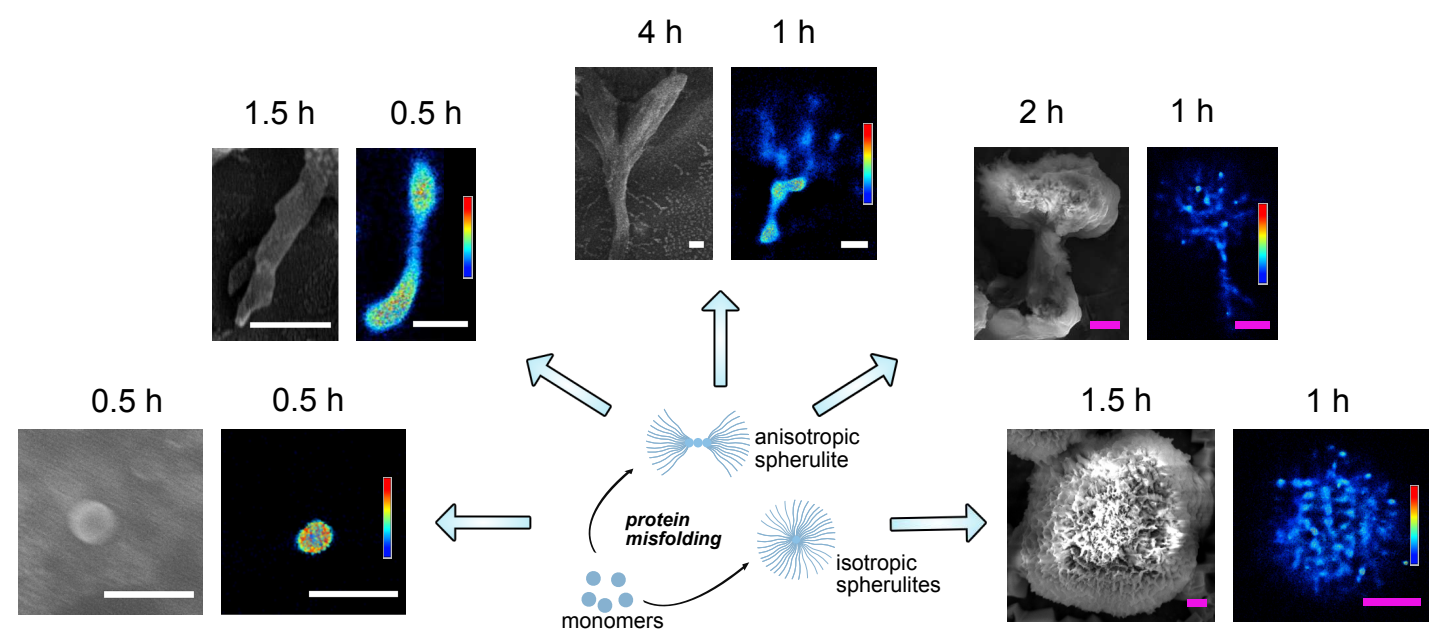

Figure 1. SEM and super-resolution 3D dSTORM reconstructed images of the co-existing in solution morphologies of the anisotropically/isotropically grown structures of different HI aggregates. 3D dSTORM images: density plots, pseudocolor scale corresponds to neighbor localisations: the density of neighboring events within a 100-nm radius sphere from localization. Pseudocolor scale ranges from 0 to 1000 for the first image on left and 0 to 400 for the rest. Scale bars in white color are $1 \mu \mathrm{m}$ and scale bars in purple color are $5 \mu \mathrm{m}$.

The diameter of the early linear aggregates increased as a function of time (Table S1). This 135 indicates that the growth was not limited to end-to-end attachment to the linear aggregate, and 136 lateral aggregation also took place. While this is to a certain extent expected ${ }^{20}$, the super resolution 137 recordings allowed its quantification. The early central linear structures, with diameters of $400 \pm$ $138100 \mathrm{~nm}$ (see Table S1), successively branched to form radially oriented amyloid fiber-like 139 structures. The further away from the core, the higher the increase in branching frequency, yielding 140 more space-filling patterns. The dimensions of the corona-like structure were $\sim 2 \mu \mathrm{m}$ to $>20 \mu \mathrm{m}$, 141 as shown in Figure 1.

142 To exclude that diverse morphologies originate from electrostatic interactions with surface 143 immobilization ${ }^{15,43}$, we used spinning disk microscopy and SEM to detect the morphology of 
144 spherulites at different growth stages in solution (see Figure S5). Consistent with the data displayed 145 in Figure 1, we detect both spherulites with asymmetrically grown (Figure 2a and 2b) and 146 symmetrically grown (Figure 2c and 2d) lobes supporting (see 3D videos of Figure 2a and 2c in 147 Supplementary Movies. S1 and S2). We confirmed that the asymmetric growth was not an artifact 148 of substrate depletion, as spherulites with asymmetric lobes had already formed by 2 hours of 149 incubation (Figure 1). This suggests that growth periods of multiple rates occurred within a single 150 sample (Figure S5), which may be masked in bulk kinetics. Moreover, our data indicated the 151 possibility that growth did not occur entirely isotropically from the central core, but rather, there 152 was initially a preferential direction.
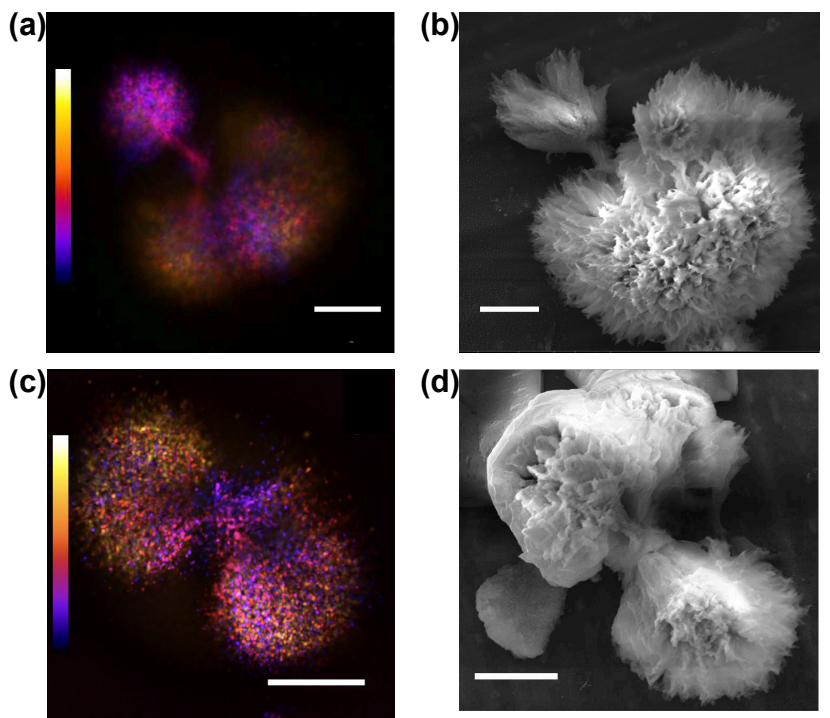

Figure 2. Structure of anisotropically grown human insulin spherulites of two distinct growth-morphologies. a) and b) Spherulites with two asymmetric sides captured by spinning disk confocal microscopy and SEM, respectively. c) and d) Spherulites with symmetric two side structures captured by spinning disk confocal microscopy and SEM, respectively. Data in (a) and (c) were acquired for a sample from incubation time of $16 \mathrm{~h}$ at $60^{\circ} \mathrm{C}$. Data in (b) and (d) are for a sample from an incubation time of $4 \mathrm{~h}$ at $60{ }^{\circ} \mathrm{C}$. Color scales are from $-14.04 \mu \mathrm{m}$ to $14.04 \mu \mathrm{m}$ in (a) and $-8.46 \mu \mathrm{m}$ to $8.46 \mu \mathrm{m}$ in (c). Scale bars are $10 \mu \mathrm{m}$. All samples were covalently labeled with Alexa Fluor 647.

\section{REPLOM: a super resolution method for the real time direct observation of growth of}

163 We developed a new super-resolution experimental method based on single-molecule 164 localisation microscopy, to quantitatively measure the growth rates at the single-aggregate level 
165 while simultaneously monitoring the morphological development of the structure. We named the 166 method REal-time kinetics via binding and Photobleaching LOcalisation Microscopy (REPLOM), 167 as it allows researchers, for the first time, to directly image the morphological development of each 168 individual aggregates in real-time with super-resolution and, simultaneously, access the kinetic 169 traces for thermodynamic analysis of the process. To perform REPLOM, HI monomers were 170 covalently labeled with Alexa Fluor 647 NHS Ester (see Supplementary Information for 171 experimental details). Figure 3a illustrates how REPLOM works: initially, only small protein 172 condensates, i.e., cores, are formed and bind to the poly-L-lysine-covered surface. The spatial 173 location of each of the fluorophores is accurately detected prior to their photobleaching ${ }^{44,45}$. 174 Optimizing the imaging settings and the absence of imaging buffer ensures rapid chromophore 175 bleaching after binding (see Methods and Figure S8). As the growth progresses, additional HI 176 monomers from the solution bind to the core, extending the dimensions of the aggregate. Each 177 labeled insulin binding event results in a diffraction-limited spot, the precise location of which can 178 be accurately extracted, similarly to in photoactivated localization microscopy (PALM) 179 methodologies ${ }^{46}$ (see Methods and Figure S9 for resolution of the method and Supplementary 180 Movie S3-S4).

181 Parallelized recordings of the spatially distinct binding of multiple individual HI loaded with 182 emitters allow the real-time direct observation of the temporal morphological development of each 183 aggregate (see Figure 3b, Figure S10, and Supplementary Movies, S3-S4). Due to the slow kinetics 184 of spherulite formation, the waiting time between each frame was 20-40 seconds, which allowed 185 to capture both seed formation and extract the growth rate of insulin aggregates. Faster frame rates 186 such as $20 \mathrm{~ms}$ are possible for the fast grown aggregates. The methodology is reliant on the intrinsic 187 bleaching of chromophores to extract their coordinates ${ }^{47-49}$ and is similar to Binding Activation 188 Localisation Microscopy (BALM) ${ }^{50}$, which measures existing structures, but additionally 189 facilitates real-time direct observation of the growth process. It also extends beyond recent 190 methods based on conventional TIRF to observe exclusively fibril growth ${ }^{20}$ or low temporal 191 resolution time lapses of linear or spherulite growth ${ }^{15,19,21}$, offering in addition rate recording and 192 morphological development of both fibrillar and spherulite structures even below the diffraction 193 limit. Consequently, the geometry and morphological development of each aggregate can be 
194 observed directly with sub-diffraction resolution, offering the extraction of each particle's growth

195 kinetics.
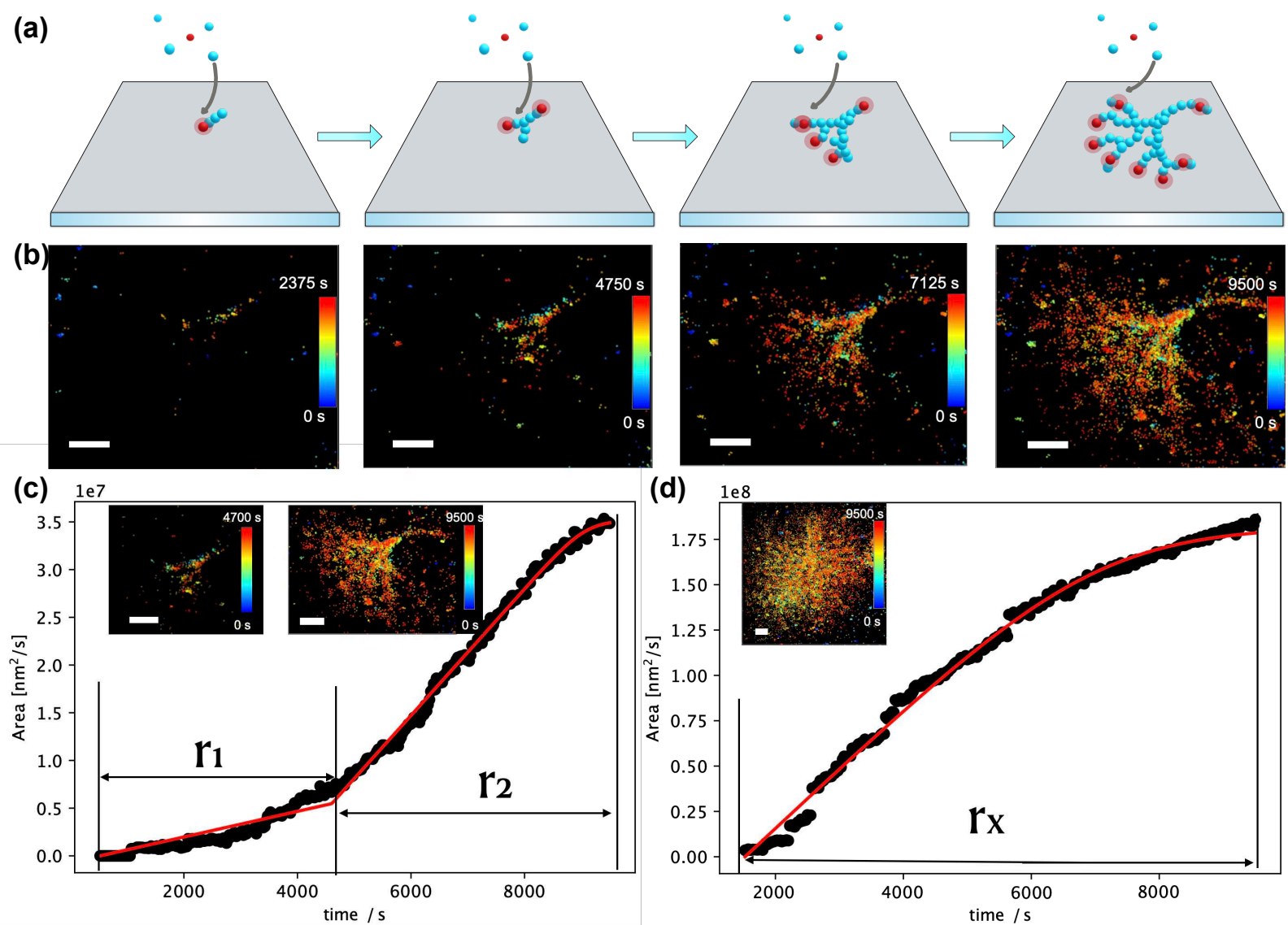

Figure 3. Direct real-time observation of HI aggregate growth by REPLOM (real-time kinetics via binding and photobleaching localisation microscopy). a) Cartoon representation of REPLOM: initially, the fluorescent signal from the small fluorescently labeled protein condensates was detected, followed by chromophore photobleaching. As the growth progressed, labeled insulins from solution bound to the aggregate, increasing the dimensions. Each binding event resulted in a diffraction-limited spot, the coordinates of which were accurately extracted, before it was photobleached by the intense laser. Parallelized recordings of the spatially distinct binding of multiple individual emitters revealed the temporal morphological development of several aggregates (Red is Alexa Fluor 647-labeled HI in fluorescent state, and blue is un-labeled insulin or Alexa Fluor 647-labeled HI in dark/photobleached state). $\mathrm{b}$ ) Direct real-time observation of temporal development of anisotropic growth at $\mathrm{t}=$ 2375 time intervals. Scale bars: $2 \mu \mathrm{m}$. c) and d) Growth curves of anisotropic spherulite (c) and isotropic spherulite (d). For anisotropic spherulites, the curve contains two parts roughly correlating with the formation of the core/linear part and branching part (see method REPLOM section). Isotropic spherulite growth was linear and followed by saturation. Inset: the corresponding HI spherulite obtained by REPLOM. Scale bars: $2 \mu \mathrm{m}$. See SI for the movies.

\section{Extraction of growth rates for diverse aggregate morphologies}


212 Consistent with the 3D dSTORM data, the direct observation of HI spherulite growth by

213 REPLOM confirmed that HI spherulites grow both anisotropically and isotropically (Figure 3). To

214 extract the growth rate kinetics for each individual aggregate, we identified the points belonging

215 to the growing aggregate with an approximate Euclidean Minimum Spanning tree segmentation ${ }^{51}$

216 and estimated the area using a Gaussian mixture model based on hierarchical clustering in Figure

$2173 \mathrm{c}$ and $3 \mathrm{~d}$ (see Supporting Information for the details) ${ }^{27-30}$. For isotropic morphologies, a single

218 linear growth rate was observed $\left(r_{x}\right)$ followed by a plateau (see Supporting Information), while for

219 anisotropic morphologies the growth curve consisted of two rate components $\left(r_{1}\right.$ and $\left.r_{2}\right)$, as shown

220 in Figure $3 \mathrm{c}$ and $3 \mathrm{~d}$ and Figure $\mathrm{S} 11 ; \mathrm{r}_{1}$ corresponds to the initial linear core and $\mathrm{r}_{2}$ to the branching

221 part, and they best fitted to reaction-limited linear growth and a diffusion-limited sigmoidal

222 growth, respectively 14,16,52-54 (see Methods and Supplementary Movies S3-S8). Consequently, the

223 growth rates $\left(r_{1}, r_{2}\right.$, and $\left.r_{x}\right)$ for each individual aggregate were extracted. The growth readouts of

224 the individual geometrically distinct morphologies allowed us to go beyond the standard analysis

225 of sigmoidal curves, which does not yield information on, or discriminate between, the temporal

226 developments for each morphology. REPLOM revealed that the anisotropic growth operated via a

227 two-step process imposed by the geometry of the growth - a pattern masked in current super-

228 resolution and bulk readouts.
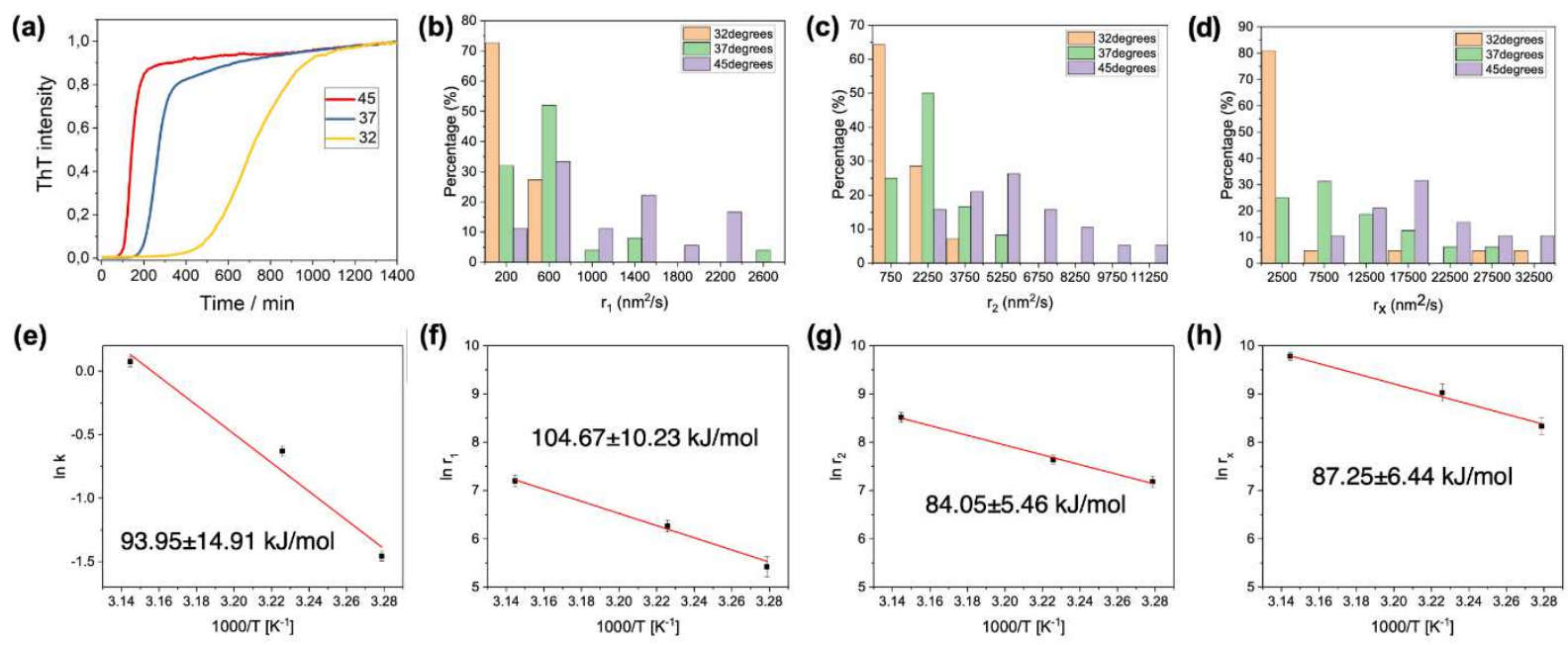

230 Figure 4. Kinetic and thermodynamic characterization of insulin aggregation a) Normalized bulk ThT distribution of anisotropic aggregates at the three different incubation temperatures: (b) linear part and (c) branching part. d) Rate distribution of isotropic aggregates at the three different incubation temperatures. e-h) Arrenhius plots 
for spherulites obtained from bulk experiments (e), and REPLOM (f-h). The formation of linear (f) and branched (g) parts of anisotropic spherulites, and the formation of isotropic spherulites (h).

Extraction of Energy barriers for the growth of diverse HI spherulites morphologies

The real-time single-particle readout from REPLOM facilitates the kinetic analysis of the temperature dependence of growth for each diffraction limited type of spherulite morphology and, consequently, the extraction of the activation energy barriers for both the spherulite morphologies and growth phase. Therefore, HI aggregate formation was induced at three different temperatures accessible without introducing optical artefacts in our microscopy setup: $45^{\circ} \mathrm{C}, 37{ }^{\circ} \mathrm{C}$, and $32{ }^{\circ} \mathrm{C}$. The ThT fluorescence measurements at the three temperatures representing the average growth kinetics are shown in Figure 4a. The rate distributions at the three temperatures for each type of morphological growth are shown in Figure 4 b, $4 \mathrm{c}$, and $4 \mathrm{~d}(\mathrm{~N}=\sim 20$, see also Figure $\mathrm{S} 12)$. As expected, the linear parts $r_{1}$ (Figure $4 b$ ) and branched parts $r_{2}$ (Figure $4 c$ ), as well as the isotropic growth rate $r_{x}$ (Figure $4 d$ ), increased at increased incubation temperature. The data do not show a pronounced curvature, and this may be due to the narrow temperature range investigated in our study and is in agreement with earlier studies ${ }^{55}$. This would suggest that the differences in heat capacity between the soluble states of the proteins and the transition states for aggregation are small ${ }^{55}$. Using the Arrhenius equation ${ }^{55,56}$ (Figure 4e-4h), we extracted the activation energy of each of the isotropic or anisotropic morphological growths and the respective linear or branching part of the individual aggregates. For the linear part of the anisotropic spherulites, the activation energy was $104.67 \pm 10.23 \mathrm{~kJ} / \mathrm{mol}$ (Figure 4f), while for the branched part it was $84.05 \pm 5.46$ $\mathrm{kJ} / \mathrm{mol}$ (Figure $4 \mathrm{~g}$ ), and for the isotropically grown spherulites it was $87.25 \pm 6.44 \mathrm{~kJ} / \mathrm{mol}$ (Figure 4h). The activation energy extracted from the bulk kinetics shown in Figure 4e (93.95 \pm 14.91 $\mathrm{kJ} / \mathrm{mol})$ is consistent with data on bovine insulin fibril formation $(\sim 100 \mathrm{~kJ} / \mathrm{mol})^{55}$. The REPLOM methodology on the other hand allowed deconvolution of a higher barrier related to step 1 in the anisotropic growth $\left(\mathrm{r}_{1}\right)$ and lower barrier in the branching part of isotropic and anisotropic growth $\left(r_{x}\right.$ and $r_{2}$ ). Together, these data indicate that the pronounced heterogeneity of growth mechanisms and structures within the aggregation ensemble leads to heterogeneity of the activation barriers.

262 We indeed highlighted that spherulite growth may proceed both isotropically and anisotropically, 263 with the latter presenting a two-step process imposed by the geometry of the growth and 

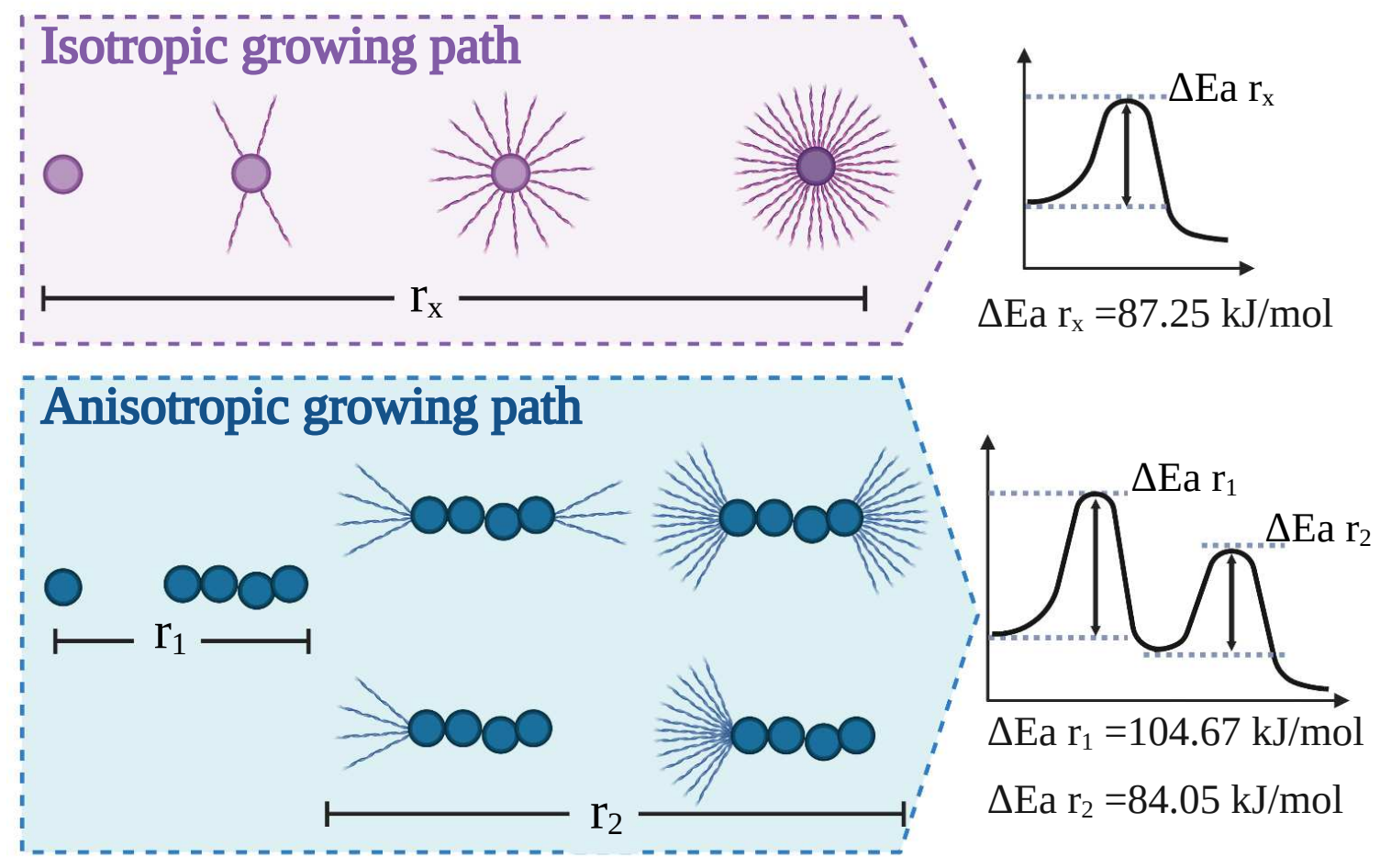

Figure 5. Schematic representation of the diverse pathways of insulin aggregation and their respective energy barriers Top: Isotropic spherulite growth, where fibril-like filaments isotropically and radially grow on a dense core. Process is characterized by a single activation energy of $\sim 87 \mathrm{~kJ} / \mathrm{mol}$. Bottom: anisotropic growth, where the dense core is growing linearly before it successively branches to form radially oriented amyloid fiber-like structures. The further the branching from the core, the more increased the branching frequency, yielding a more space-filling pattern. The process involves two steps imposed by the geometry of the growth and characterized by two activation energies of 104 and $84 \mathrm{~kJ} / \mathrm{mol}$ for the linear and branching parts, respectively.

\section{Conclusions}

276 Our combined results revealed that the growth of amyloid core-shell structures for insulin, i.e.,

277 spherulites, may proceed not only via isotropic growth but also by following a multistep pathway 278 characterized by initial pronounced anisotropic behavior (Figure 5). The anisotropic growth may 279 thus not be an exclusive property of metal alloys, salts and minerals, but may extend to protein 280 aggregates. In essence are data are consistent with a unifying mechanism underlying chemical 281 growth of both biological soft materials and hard-non biological composites. Such variability in 282 growth within the same aggregation reaction results in a spectrum of aggregation kinetics traces 
283 that can be quantitatively detected by our method, allowing the operator to extract the 284 thermodynamic parameters for each of the aggregation subsets. These findings underscore how 285 conclusions solely based on bulk kinetics data may overlook the complexity and heterogeneity of 286 the aggregation process.

287 Our novel experimental approach offers real-time detection of super-resolution images during 288 protein aggregation kinetics. The REPLOM method allows the direct observation of self-assembly 289 kinetics at the level of single aggregates and the quantification of the heterogeneity of aggregates 290 and their growth mechanisms, which are otherwise masked with current methodologies. Our 291 general framework can be extended to the simultaneous detection of markedly different structures 292 within a single aggregation reaction and contribute to research into a more comprehensive 293 representation of the generalized energy landscape of proteins. This will offer the unique 294 possibility of disentangling different mechanisms leading to the myriad of aggregate structures 295 that occur. The method is implemented on the insulin model systems, but can be easily translatable 296 to more medically relevant proteins, such as $\alpha$-synuclein or A $\beta$ peptide. Deciphering whether these 297 structures persist in the context of the cellular environment and the direct physiological 298 implications of anisotropically grown morphologies would require combination of our 299 methodologies with DNA-paint and antibodies as recently developed ${ }^{57}$. Our approach may indeed 300 provide unprecedented information on transient intermediate species, which are nowadays 301 recognized as the cause of progression in many diseases, in terms of both energetics and 302 morphology. Finally, our approach is general and may be applicable to generic self-assembly 303 reactions of systems characterized by a high degree of heterogeneity.

\section{Methods-Microscopy}

\section{Spinning Disk Microscopy.}

306 The 3D images of grown spherulites were taken by a SpinSR10-spinning disk confocal super 307 resolution microscope (Olympus) using a silicone oil-immersion 100x objective 308 (UPLSAPO100XS, NA=1.35, Olympus). The Alexa Fluor 647-labeled HI spherulites were excited 309 with a $640 \mathrm{~nm}$ laser (OBIS COHERENT). The exposure time was $50 \mathrm{~ms}$ and the z step length was $310 \quad 0.36 \mu \mathrm{m}$. 


\section{Scanning Electron Microscopy (SEM).}

312 SEM images of spherulites were taken by using a Quanta FEG 200 ESEM microscope.

\section{Cross Polarized Microscopy.}

314 Images were collected using a 10x objective and crossed polarised which enabled spherulites to 315 show the characteristic Maltese cross (Zeiss Axioplan Optical Microscope, Carl Zeiss).

\section{Super-resolution Imaging}

317 Super resolution imaging was attained on an inverted Total Internal Reflecion microscope (TIRF) 318 (Olympus IX-83) with a 100x oil immersion objective (UAPON 100XOTIRF, NA=1.49, 319 Olympus) Alexa Fluor 647 was excited by a $640 \mathrm{~nm}$ solid state laser line (Olympus) and reflected 320 to a quad band filter cube (dichroic mirrors ZT640rdc, ZT488rdc and ZT532rdc for splitting and 321 with single-band bandpass filters FF02-482/18-25, FF01-532/3-25 and FF01-640/14-25). Signal 322 was detected by an EMCCD camera (imagEM X2, Hamamatsu).

\section{D direct Stochastic Optical Reconstruction Microscopy (3D dSTORM) and image}

324 analysis.

325 3D dSTORM imaging was achieved by installing a cylindrical lens $(\mathrm{f}=500 \mathrm{~mm})$ in the emission 326 pathway of (TIRF) to introduce the astigmatism of point spread function (PSF) ${ }^{32}$. All the

327 dSTORM imaging experiments were performed at room temperature $\left(21^{\circ} \mathrm{C}\right)$. The exposure time 328 was $30 \mathrm{~ms}$ and 10000 frames for each movie.

329 To extract $\mathrm{z}$ information from the widths of single molecule images, we generated a calibration 330 curve of PSF width in the lateral plane (Wx and Wy) as a function of height by measuring Atto 331 655-labeled liposomes using TIRF with a step size of $10 \mathrm{~nm}$ and exposure time of $30 \mathrm{~ms}$ (Fig. S3).

332 The $\mathrm{HI}$ aggregates which were incubated in a block heater for 0.5 hours to 2 hours at $60{ }^{\circ} \mathrm{C}$. At the 333 desired time they were added to the poly-L-Lysine treated microscope chamber ${ }^{58}$ and incubated 334 for $10 \mathrm{~min}$ at room temperature to ensure immobilization. Extra sample was washed away with 335 MilliQ water. Imaging buffer containing $50 \mathrm{mM}$ Tris, $10 \mathrm{mM} \mathrm{NaCl}, 10 \%$ (w/v) glucose, 0.5 $336 \mathrm{mg} / \mathrm{mL}$ glucose oxidase, $40 \mu \mathrm{g} / \mathrm{mL}$ catalase and 0.1 M MEA ${ }^{59}$ was flushed into the chamber for 337 dSTORM imaging. All measurements were carried out at room temperature. The optimal ratio of 338 labeled to unlabeled insulin that provided reliable signal without affecting the aggregation process or compromising resolution was 1 to 60,000 . This is quite different from earlier dSTORM imaging 
340 of fibrils using a ratio of $1 / 20^{24}$ because of the much higher 3D density of spherulites that prevent 341 reliable super resolution imaging at high labeling ratios.

342 The 3D dSTORM data was analysed by ThunderSTORM ${ }^{60}$. The $\mathrm{z}$ information of individual 343 localisations was extracted based on the calibration curves (calculated by ThunderSTORM, shown 344 in Figure S3). The detected localisations were further filtered according to their intensity and drift 345 correction, in order to remove some possible false positive or poor quality detections. 3D super346 resolution images were visualized with ViSP software ${ }^{61}$.

\section{REal-time kinetic via Photobleaching Localisation Microscopy (REPLOM)}

348 Preparation of HL aggregates and imaging. The solution containing $5 \mathrm{mg} / \mathrm{mL} \mathrm{HI}$ monomer 349 was first incubated in a block heater to skip the lag phase. The optimal pre-incubation time for 350 spherulite formation on the microscope surface was found to be $\sim 8$ hours for $45^{\circ} \mathrm{C}, 20$ hours for $35137^{\circ} \mathrm{C}$ and 75 hours for $32^{\circ} \mathrm{C}$, respectively. Then they were transferred to poly-L-lysine coated 352 glass slide chambers and covered by a lip to prevent solvent evaporation during imaging (Figure 353 S6).

354 REPLOM was performed on the same TIRF microscope setup as the 3D STORM without the 355 cylindrical lens. Alexa Fluor 647 labeled HI was excited by 640nm solid state laser lines 356 (Olympus). We found the optimal ratio of labeled to unlabeled insulin for REPLOM to be ca. 1 to 357 10,000. A high labelling density would result in proximate fluorophores from the newly grown 358 area emitting simultaneously and therefore cause mislocalization ${ }^{24}$. Too low labeling ratio may 359 cause some details, e.g. small branching part, during spherulites growth to be undetected. Imaging 360 was performed with an exposure time of $30 \mathrm{~ms}$ followed by a waiting time for each frame of 2036140 seconds so as to capture in real time the slow kinetics of spherulite formation. This frame rate 362 allowed to capture both seed formation and extract the growth rate of insulin aggregates. Faster 363 frame rates may be required for different protein aggregates ${ }^{62}$. All image acquisition was 364 performed at the same incubation temperatures as in the block heater. The incubation temperatures 365 during the imaging processes were achieved by a heating unit 2000 (PECON).

366 Data analysis. The data was analysed by ThunderSTORM. Some possible false positive or poor367 quality detections were removed by intensity filter. Figure S7 shows the comparison of images 
prior to and after drift correction. The reconstructed images with time series were obtained by ViSP ${ }^{61}$ software. For Quantification of growth kinetics is available in Supporting Information.

Lifetime of fluorophores. The lifetime of fluorophores in REPLOM was evaluated by checking the duration time of fluorescent state before they were photobleached. We checked 1885 individual

372 Alexa Fluor 647 fluorophores and found they were photobleached very fast without imaging buffer 373 (Figure S7). The lifetime is about $0.7845 \pm 0.0017$ frames.

Resolution of REPLOM. The resolution of REPLOM was determined by the FWHM of single spot's intensity (Figure S9) using an adapted version of previously published software ${ }^{45,63}$. Briefly, using our subpixel resolution software, we were able to extract multiple (91) single spots (see Figure S9) and align all to the same center. Fitting a two-dimensional gaussian to the resulting stacked clusters allowed the reliable extraction of FWHM used to determine the obtained resolution. Using a maximum likelihood fitting scheme avoided potential bias from data binning. identification of candidates for fluorophores docking on a growing aggregate was inspired by recent published work ${ }^{51}$ and done in the following way:

383 First, using all detected RE-PLOM spots from the movie, an approximate Euclidean Minimum 384 Spanning tree was constructed using only the 30 nearest neighbors as candidates for edges. 385 Regions of aggregate candidates were cut from each other by removing all edges with lengths more than the 95th percentile. This is an effective way of separating high-density regions from lowdensity regions. The computation was done using the function HierarchicalClustering from the astroML python package. Since we were interested mostly in the large insulin aggregates where the internal structure was visible, it was decided that all clusters obtained in this manner with less than 100 detected fluorophores were excluded from the subsequent analysis.

391 The time-dependency of the aggregate growth was found by a similar approach. At each frame, 392 for a cluster, a refined grouping was done by cutting an approximate Euclidean Minimum 393 Spanning tree made using 10 neighbors with a distance cutoff of 400nm which was found to be 394 optimal for removal of most spots outside the aggregate while still not cutting up the main group. 395 The points from the largest subgroup resulting from this analysis were defined to be the aggregate 396 for that frame. 
The area of the aggregate was estimated using a gaussian mixture model with a component for 398 every 5 points in the aggregate, but not less than 25 components ${ }^{51}$. We defined the area of the 399 aggregate as the region lying above the average probability density in this fit. The growth profile 400 resulting from our approach had a few artifacts like jumps and fluctuations due to mixture model 401 fitting and aggregate segmentation, but we found that the resulting growth curve in most cases had 402 an identifiable trend, and the results were quite consistent across parameter choices.

403 From the estimated area of the aggregate in each frame, a growth curve could be plotted.

404 The radial growth rate of such aggregates has previously been found to be either reaction-limited 405 or diffusion-limited, leading to linear increase in time or increase as $\propto \sqrt{t}$ respectively ${ }^{14,52-54}$. If 406 we assume that the estimated area of the aggregated is directly related to the radius as $A \propto R^{2}$ the 407 two growth types lead to the following models

$$
\frac{\mathrm{d} A(t)}{\mathrm{d} t}=r_{1},
$$

$$
\frac{\mathrm{d} A(t)}{\mathrm{d} t}=\frac{1}{2} r_{1} t .
$$

413 Where the first model is diffusion limited and the second is reaction limited. We found that many 414 of the structures where initially consistent with reaction limited diffusion and then shifted to either 415 diffusion or reaction limited growth with a new rate. To allow for this shift, we let the growth be 416 diffusion limited up to a switch-point $t_{0}$ after which the growth rate changes. We formulate one 417 such model which ends reaction limited and one which remains diffusion limited

$$
\begin{gathered}
\frac{\mathrm{d} A(t)}{\mathrm{d} t}=\left\{\begin{array}{ll}
r_{1}, & t_{0}>t \\
r_{2}, & t_{0} \leq t
\end{array},\right. \\
\frac{\mathrm{d} A(t)}{\mathrm{d} t}=\left\{\begin{array}{ll}
r_{1}, & t_{0}>t \\
\frac{1}{2} r_{2} t, & t_{0} \leq t
\end{array} .\right.
\end{gathered}
$$


422 Finally, without continuous flow of constituent monomer, the growth inevitably saturates at a 423 plateau ${ }^{16}$. For both models, we therefore introduce a switch time $t_{1}$ after which the growth slowly 424 saturates sigmoidally over a time interval $5 \tau$

425

$$
\frac{\mathrm{d} A_{\operatorname{lin}}(t)}{\mathrm{d} t}=\left\{\begin{array}{c}
r_{1}, \quad t_{0}>t \\
r_{2}, t_{0} \leq t<t_{1} \\
r_{2} \frac{1}{1+e^{\frac{5\left(t-\tau-t_{1}\right)}{\tau}},} t_{1} \leq t
\end{array},\right.
$$

$$
\frac{\mathrm{d} A_{\mathrm{par}}(t)}{\mathrm{d} t}=\left\{\begin{array}{c}
r_{1}, \quad t_{0}>t \\
\frac{1}{2} r_{2} t, \quad t_{0} \leq t<t_{1} \\
\frac{1}{2} r_{2} \frac{t}{1+e^{\frac{5\left(t-\tau-t_{1}\right)}{\tau}}}, \quad t_{1} \leq t
\end{array}\right.
$$

430 Where we introduced the names $A_{\text {lin }}$ and $A_{\text {par }}$ referring to the linear-like and parabolic-like shape 431 of the two resulting growth curves. We found the anisotropic spherulites to fit best with $A_{\text {lin }}$ and 432 the isotropic spherulites fit best with $A_{\text {par. }}$

433 When fitting an experimentally observed aggregate growth curve $\left\{A_{i}, t_{i}\right\}, i \in(0, N-1)$ the 434 equations where numerically integrated from an initial timepoint $\left(A_{0}, t_{0}\right)$ to the final timepoint $435\left(A_{N-1}, t_{N-1}\right)$. For each growth curve, the parameters $\left(r_{1}, r_{2}, t_{0}, t_{1}, \tau\right)$ were estimated with a chi2 436 fit. Each fit was run twice, the first fit was unweighted and were used to estimate the error bars 437 using the standard deviation of the residuals. The second fit used the residuals in a weighted chi2 438 fit to obtain the final fit parameters for the growth curve.

\section{Data availability}


441 All data sets used for figures are provided as source data in the manuscript. Source code and 442 executable can be found at https://github.com/hatzakislab/REPLOM-analysis-tool. All source

443 data are available at https://sid.erda.dk/sharelink/fje3exOlq2.

445 Author information

446 Corresponding Author

$447 \quad *$ Vito Foderà, Email: vito.fodera@sund.ku.dk

448 * Nikos S. Hatzakis, Email: hatzakis@chem.ku.dk (NSH).

449 Author Contributions

450 M.Z, N.S.H and V.F wrote the paper with feedback from all authors. M.Z designed, carried out 451 and analysed all microscopy experiments, and prepared all samples. H.D.P wrote the automated 452 cluster finding and rates analysis algorithm. M.Z and X.Z did the ThT-fluorescence and turbidity 453 measurements. S.S-R.B calculated the resolution of REPLOM and fluorophore's lifetime. L.B and 454 A.Z helped with the mechanism explanation. N.S.H conceived the project idea, in collaboration 455 with V.F., and had the overall project management and strategy.

$456 \quad$ Notes

457 The authors declare no competing financial interest.

\section{Acknowledgment}

459 This work was funded by the Lundbeck foundation (grant R250-2017-1293 and R346-2020-1759)

460 for M.Z. Villum foundation young investigator fellowship (grant 10099), and the Carlsberg 461 foundation Distinguished Associate professor program (CF16-0797) and the NovoNordisk Center 462 for Biopharmaceuticals and Biobarriers in Drug Delivery (NNF16OC0021948) for N.S.H. Villum 463 foundation young investigator fellowship (grant 19175), the Novo Nordisk foundation 464 (NNF16OC0021948) and Lundbeck foundation (R155-2013-14113) for V.F. China Scholarship 465 Council (201709110108) for X.Z. Work at The Novo Nordisk Foundation Center for Protein 466 Research (CPR) that NSH is associated with, is funded by a generous donation from the Novo 
Nordisk Foundation (Grant number NNF14CC0001). We thank Dr Y. Hu from Technical

468 University of Denmark for the help with SEM imaging.

469 N.S.H. and V.F are members of the Integrative Structural Biology Cluster (ISBUC) at the 470 University of Copenhagen.

\section{REFERENCES}

4721 Chiti, F. \& Dobson, C. M. Protein Misfolding, Functional Amyloid, and Human Disease.

473 Annual Review of Biochemistry 75, 333-366,

474 doi:10.1146/annurev.biochem.75.101304.123901 (2006).

4752 House, E., Jones, K. \& Exley, C. Spherulites in Human Brain Tissue are Composed of

476 Beta Sheets of Amyloid and Resemble Senile Plaques. Journal of Alzheimers Disease 25,

477

478

479

480

481

482

483 43-46, doi:10.3233/jad-2011-110071 (2011).

3 Exley, C. et al. Spherulites of Amyloid-beta(42) In Vitro and in Alzheimer's Disease. Journal of Alzheimers Disease 20, 1159-1165, doi:10.3233/jad-2010-091630 (2010).

4 Yumlu, S., Barany, R., Eriksson, M. \& Röcken, C. Localized insulin-derived amyloidosis in patients with diabetes mellitus: a case report. Human Pathology 40, 1655-1660, doi:https://doi.org/10.1016/j.humpath.2009.02.019 (2009).

5 Jiang, Y. et al. Protein Spherulites for Sustained Release of Interferon: Preparation, Characterization and in vivo Evaluation. Journal of Pharmaceutical Sciences 100, 19131922, doi:https://doi.org/10.1002/jps.22403 (2011).

6 Vetri, V. \& Foderà, V. The route to protein aggregate superstructures: Particulates and amyloid-like spherulites. FEBS Letters 589, 2448-2463, doi:http://doi.org/10.1016/j.febslet.2015.07.006 (2015).

7 Krebs, M. R. H. et al. The formation of spherulites by amyloid fibrils of bovine insulin. Proceedings of the National Academy of Sciences of the United States of America 101, 14420-14424, doi:10.1073/pnas.0405933101 (2004).

8 Lu, Z. P., Goh, T. T., Li, Y. \& Ng, S. C. Glass formation in La-based La-Al-Ni-Cu-(Co) alloys by Bridgman solidification and their glass forming ability. Acta Materialia 47, 2215-2224, doi:https://doi.org/10.1016/S1359-6454(99)00058-0 (1999).

9 Heaney, P. J. \& Davis, A. M. Observation and Origin of Self-Organized Textures in Agates. Science 269, 1562-1565, doi:10.1126/science.269.5230.1562 (1995).

10 Hosier, I. L., Bassett, D. C. \& Vaughan, A. S. Spherulitic Growth and Cellulation in Dilute Blends of Monodisperse Long n-Alkanes. Macromolecules 33, 8781-8790, doi:10.1021/ma000946t (2000).

11 Kajioka, H., Hikosaka, M., Taguchi, K. \& Toda, A. Branching and re-orientation of lamellar crystals in non-banded poly(butene-1) spherulites. Polymer 49, 1685-1692, doi:https://doi.org/10.1016/j.polymer.2008.01.066 (2008).

12 Garcia, G. A., Cohen, S. I. A., Dobson, C. M. \& Knowles, T. P. J. Nucleationconversion-polymerization reactions of biological macromolecules with prenucleation clusters. Physical Review E 89, 032712, doi:10.1103/PhysRevE.89.032712 (2014).

13 Krebs, M. R. H., Bromley, E. H. C., Rogers, S. S. \& Donald, A. M. The mechanism of amyloid spherulite formation by bovine insulin. Biophys. J. 88, 2013-2021, doi:10.1529/biophysj.104.051896 (2005). 
50914 Domike, K. R. \& Donald, A. M. Thermal Dependence of Thermally Induced Protein

$510 \quad$ Spherulite Formation and Growth: Kinetics of $\beta$-lactoglobulin and Insulin.

511 Biomacromolecules 8, 3930-3937, doi:10.1021/bm7009224 (2007).

51215 Ban, T. et al. Real-time and Single Fibril Observation of the Formation of Amyloid $\beta$

16 Domike, K. R. \& Donald, A. M. Kinetics of spherulite formation and growth: Salt and protein concentration dependence on proteins $\beta$-lactoglobulin and insulin. International Journal of Biological Macromolecules 44, 301-310, doi:https://doi.org/10.1016/j.ijbiomac.2008.12.014 (2009).

17 Foderà, V.\& Donald, A. M. Tracking the heterogeneous distribution of amyloid spherulites and their population balance with free fibrils. The European Physical Journal E 33, 273-282, doi:10.1140/epje/i2010-10665-4 (2010).

18 Toprakcioglu, Z., Challa, P., Xu, C. \& Knowles, T. P. J. Label-Free Analysis of Protein Aggregation and Phase Behavior. ACS Nano 13, 13940-13948, doi:10.1021/acsnano.9b05552 (2019).

19 Yagi, H., Ban, T., Morigaki, K., Naiki, H. \& Goto, Y. Visualization and Classification of Amyloid $\beta$ Supramolecular Assemblies. Biochemistry 46, 15009-15017, doi:10.1021/bi701842n (2007).

20 Zimmermann, M. R. et al. Mechanism of Secondary Nucleation at the Single Fibril Level from Direct Observations of A $\beta 42$ Aggregation. Journal of the American Chemical Society 143, 16621-16629, doi:10.1021/jacs.1c07228 (2021).

21 Andersen, C. B. et al. Branching in Amyloid Fibril Growth. Biophysical Journal 96, 1529-1536, doi:http://doi.org/10.1016/j.bpj.2008.11.024 (2009).

22 Vetri, V.et al. Ethanol Controls the Self-Assembly and Mesoscopic Properties of Human Insulin Amyloid Spherulites. The Journal of Physical Chemistry B 122, 3101-3112, doi:10.1021/acs.jpcb.8b01779 (2018).

23 Johansson, P. K. \& Koelsch, P. Label-free imaging of amyloids using their intrinsic linear and nonlinear optical properties. Biomed. Opt. Express 8, 743-756, doi:10.1364/BOE.8.000743 (2017).

24 Pinotsi, D. et al. Direct Observation of Heterogeneous Amyloid Fibril Growth Kinetics via Two-Color Super-Resolution Microscopy. Nano Letters 14, 339-345, doi:10.1021/nl4041093 (2014).

25 Cox, H., Georgiades, P., Xu, H., Waigh, T. A. \& Lu, J. R. Self-Assembly of Mesoscopic Peptide Surfactant Fibrils Investigated by STORM Super-Resolution Fluorescence Microscopy. Biomacromolecules 18, 3481-3491, doi:10.1021/acs.biomac.7b00465 (2017).

26 Kaminski Schierle, G. S. et al. In Situ Measurements of the Formation and Morphology of Intracellular $\beta$-Amyloid Fibrils by Super-Resolution Fluorescence Imaging. Journal of the American Chemical Society 133, 12902-12905, doi:10.1021/ja201651w (2011).

27 Pinholt, H. D., Bohr, S. S.-R., Iversen, J. F., Boomsma, W. \& Hatzakis, N. S. Singleparticle diffusional fingerprinting: A machine-learning framework for quantitative analysis of heterogeneous diffusion. Proceedings of the National Academy of Sciences 118, e2104624118, doi:10.1073/pnas.2104624118 (2021).

28 Thomsen, J. et al. DeepFRET, a software for rapid and automated single-molecule FRET data classification using deep learning. eLife 9, doi:10.7554/eLife.60404 (2020). 
29 Stella, S. et al. Conformational Activation Promotes CRISPR-Cas12a Catalysis and Resetting of the Endonuclease Activity. Cell 175, 1856-1871.e1821, doi:https://doi.org/10.1016/j.cell.2018.10.045 (2018).

30 Jensen, S. B. et al. Biased cytochrome P450-mediated metabolism via small-molecule ligands binding P450 oxidoreductase. Nat. Commun. 12, 2260, doi:10.1038/s41467-02122562-w (2021).

31 Malle, M. G. et al. Single particle combinatorial multiplexed liposome fusion mediated by DNA. bioRxiv, 2021.2001.2019.427313, doi:10.1101/2021.01.19.427313 (2021).

32 Huang, B., Wang, W., Bates, M. \& Zhuang, X. Three-Dimensional Super-Resolution Imaging by Stochastic Optical Reconstruction Microscopy. Science 319, 810-813, doi:10.1126/science.1153529 (2008).

33 Hayashi, S. \& Okada, Y. Ultrafast superresolution fluorescence imaging with spinning disk confocal microscope optics. Molecular Biology of the Cell 26, 1743-1751, doi:10.1091/mbc.E14-08-1287 (2015).

34 Foderà, V., van de Weert, M. \& Vestergaard, B. Large-scale polymorphism and autocatalytic effect in insulin fibrillogenesis. Soft Matter 6, 4413-4419, doi:10.1039/C0SM00169D (2010).

35 Foderà, V. et al. Self-Organization Pathways and Spatial Heterogeneity in Insulin Amyloid Fibril Formation. The Journal of Physical Chemistry B 113, 10830-10837, doi:10.1021/jp810972y (2009).

36 Foderà, V., Zaccone, A., Lattuada, M. \& Donald, A. M. Electrostatics Controls the Formation of Amyloid Superstructures in Protein Aggregation. Physical Review Letters 111, 108105, doi:10.1103/PhysRevLett.111.108105 (2013).

37 Rogers, S. S., Krebs, M. R. H., Bromley, E. H. C., van der Linden, E. \& Donald, A. M. Optical Microscopy of Growing Insulin Amyloid Spherulites on Surfaces In Vitro. Biophysical Journal 90, 1043-1054, doi:10.1529/biophysj.105.072660 (2006).

38 Shtukenberg, A. G., Punin, Y. O., Gunn, E. \& Kahr, B. Spherulites. Chemical Reviews 112, 1805-1838, doi:10.1021/cr200297f (2012).

39 Gránásy, L., Pusztai, T., Tegze, G., Warren, J. A. \& Douglas, J. F. Growth and form of spherulites. Physical Review E 72, 011605, doi:10.1103/PhysRevE.72.011605 (2005).

40 Shen, Y. et al. Biomolecular condensates undergo a generic shear-mediated liquid-tosolid transition. Nature Nanotechnology 15, 841-847, doi:10.1038/s41565-020-0731-4 (2020).

41 Song, S. et al. Spherulite-like Micelles. Angewandte Chemie International Edition n/a, doi:https://doi.org/10.1002/anie.202101177.

42 Galkin, O.\& Vekilov, P. G. Mechanisms of Homogeneous Nucleation of Polymers of Sickle Cell Anemia Hemoglobin in Deoxy State. Journal of Molecular Biology 336, 4359, doi:https://doi.org/10.1016/j.jmb.2003.12.019 (2004).

43 Elsharkawy, S. et al. Protein disorder-order interplay to guide the growth of hierarchical mineralized structures. Nature Communications 9, doi:ARTN 214510.1038/s41467-01804319-0 (2018).

44 Moses, M. E. et al. Single-Molecule Study of Thermomyces lanuginosus Lipase in a Detergency Application System Reveals Diffusion Pattern Remodeling by Surfactants and Calcium. ACS Appl. Mater. Interfaces 13, 33704-33712, doi:10.1021/acsami.1c08809 (2021). 
60045 Bohr, S. S. R. et al. Direct observation of Thermomyces lanuginosus lipase diffusional states by Single Particle Tracking and their remodeling by mutations and inhibition. Sci Rep-Uk 9, 16169, doi:10.1038/s41598-019-52539-1 (2019).

604 Betzig, E. et al. Imaging Intracellular Fluorescent Proteins at Nanometer Resolution. Science 313, 1642-1645, doi:10.1126/science.1127344 (2006).

Gordon, M. P., Ha, T. \& Selvin, P. R. Single-molecule high-resolution imaging with photobleaching. Proceedings of the National Academy of Sciences of the United States of America 101, 6462-6465, doi:10.1073/pnas.0401638101 (2004). Qu, X., Wu, D., Mets, L. \& Scherer, N. F. Nanometer-localized multiple single-molecule fluorescence microscopy. Proceedings of the National Academy of Sciences of the United States of America 101, 11298-11303, doi:10.1073/pnas.0402155101 (2004).

\section{Burnette, D. T., Sengupta, P., Dai, Y., Lippincott-Schwartz, J. \& Kachar, B.} Bleaching/blinking assisted localization microscopy for superresolution imaging using standard fluorescent molecules. Proceedings of the National Academy of Sciences 108, 21081-21086, doi:10.1073/pnas.1117430109 (2011).

50 Ries, J. et al. Superresolution Imaging of Amyloid Fibrils with Binding-Activated Probes. ACS Chemical Neuroscience 4, 1057-1061, doi:10.1021/cn400091m (2013). Cowan, N. B. \& Ivezić, Ž. The Environment of Galaxies at Low Redshift. The Astrophysical Journal 674, L13-L16, doi:10.1086/528986 (2008).

\section{8, doi:https://doi.org/10.1016/0022-0248(87)90051-0 (1987).}

53 Majumder, S., Busch, H., Poudel, P., Mecking, S. \& Reiter, G. Growth Kinetics of Stacks of Lamellar Polymer Crystals. Macromolecules 51, 8738-8745, doi:10.1021/acs.macromol.8b01765 (2018).

54 Tanaka, H. \& Nishi, T. New Types of Phase Separation Behavior during the Crystallization Process in Polymer Blends with Phase Diagram. Physical Review Letters 55, 1102-1105, doi:10.1103/PhysRevLett.55.1102 (1985).

55 Buell, A. K. et al. Detailed Analysis of the Energy Barriers for Amyloid Fibril Growth. Angewandte Chemie International Edition 51, 5247-5251, doi:https://doi.org/10.1002/anie.201108040 (2012).

56 Cohen, S. I. A. et al. Distinct thermodynamic signatures of oligomer generation in the aggregation of the amyloid- $\beta$ peptide. Nat Chem 10, 523-531, doi:10.1038/s41557-0180023-x (2018).

57 Sang, J. C. et al. Super-resolution imaging reveals $\alpha$-synuclein seeded aggregation in SHSY5Y cells. Communications Biology 4, 613, doi:10.1038/s42003-021-02126-w (2021).

58 Chen, W. et al. Fluorescence Self-Quenching from Reporter Dyes Informs on the Structural Properties of Amyloid Clusters Formed in Vitro and in Cells. Nano Letters 17, 143-149, doi:10.1021/acs.nanolett.6b03686 (2017).

59 Huang, B., Jones, S. A., Brandenburg, B. \& Zhuang, X. Whole-cell 3D STORM reveals interactions between cellular structures with nanometer-scale resolution. Nature Methods 5, 1047, doi:10.1038/nmeth.1274

643 https://www .nature.com/articles/nmeth.1274\#supplementary-information (2008).

644

Ovesný, M., Kř́̌žek, P., Borkovec, J., Švindrych, Z. \& Hagen, G. M. ThunderSTORM: a comprehensive ImageJ plug-in for PALM and STORM data analysis and super-resolution imaging. Bioinformatics 30, 2389-2390, doi:10.1093/bioinformatics/btu202 (2014). 
$64561 \quad$ Beheiry, M. E. \& Dahan, M. ViSP: representing single-particle localizations in three 646 dimensions. Nature Methods 10, 689, doi:10.1038/nmeth.2566

647 https://www.nature.com/articles/nmeth.2566\#supplementary-information (2013).

64862 Ogi, H. et al. Ultrafast propagation of $\beta$-amyloid fibrils in oligomeric cloud. Sci Rep-Uk 649 4, 6960, doi:10.1038/srep06960 (2014).

65063 Thomsen, R. P. et al. A large size-selective DNA nanopore with sensing applications.

651 Nat. Commun. 10, 5655, doi:10.1038/s41467-019-13284-1 (2019).

652 


\section{Supplementary Files}

This is a list of supplementary files associated with this preprint. Click to download.

- REPLOMSI.pdf

- SupplementaryMovie1.mp4

- SupplementaryMovie2.mp4

- SupplementaryMovie3.mp4

- SupplementaryMovie4.mp4

- SupplementaryMovie5.mp4

- SupplementaryMovie6.mp4

- SupplementaryMovie7.mp4

- SupplementaryMovie8.mp4 